\title{
Wegener's Granulomatosis with Peripheral Eosinophilia in a Young Adult Patient
}

SUNIL KUMAR, ${ }^{1}$ S K DIWAN, ${ }^{2}$ SWAPNIL CHILLAWAR, ${ }^{3}$ SHAGUN SHABARWAL, ${ }^{3}$ SONAM GOYAL, ${ }^{3}$ SHRADDHA JAIN 4

\begin{abstract}
Wegener's granulomatosis is a rare autoimmue disease with multi-system involvement that manifests as granulomatous vasculitic lesions, mainly involving the upper respiratory tract, lower respiratory tract and kidney. We present a case in a young adult male patient presenting with complaints of epistaxis, cough and hemoptysis who was diagnosed as Wegener's granulomatosiswith peripheral eosinophilia on the basis of chest x-ray and CT Chest and pathological confirmation by high level of serum cytoplasmic antineutrophil cytoplasmic antibody.
\end{abstract}

Key words: Wegener's granulomatosis, eosinophilia, Churg- Strauss syndrome, young adult

\section{Introduction:}

Wegener's Granulomatosis (WG) was described in detail by Friedrich Wegener in 1936. WGwith polyangiitis is a distinct clinicopathologic entity characterized by granulomatous vasculitis of the upper and lower respiratory tracts together with kidney lesion. ${ }^{1}$ It is typically associated with proteinase 3 Antineutrophil cytoplasmic antibodies (PR3-ANCA), which cause a cytoplasmic (C-ANCA) pattern of staining on immunofluorescence testing of serum. The presence of PR3ANCA makes us suspect the diagnosis of WG rather than other vasculitic diseases (2). Churg-Strauss syndrome (CSS) is another type of systemic vasculitis characterized by peripheral and tissue eosinophilia. ANCA is present in more than one-half of the patients with CSS, but almost all CSS patients have MPO-ANCA (ANCA specific for myeloperoxidase) which causesperinuclear (P-ANCA) staining on immuno-fluorescence testing. ${ }^{2}$ In such a situation, it is difficult to differentiate WG from CSS. In this case report we discuss the differential diagnosis of these two types of vasculitis and the clinical characteristics of WG with eosinophilia. This case report highlights the importance of radiological investigations and systematic clinical-radiological correlation, leading to diagnosis of an uncommon condition in an unsuspecting young male patient.

1. Associate Professor, Department of Medicine, JawaharLal Nehru Medical College, DMIMSU, Sawangi, wardha, Maharastra, India.

2. Professor, Department of Medicine, JawaharLal Nehru Medical College, DMIMSU, Sawangi, wardha, Maharastra, India.

3. Resident, Department of Medicine, JawaharLal Nehru Medical College, DMIMSU, Sawangi, wardha, Maharastra, India.

4. Associate professor, Department of ENT, JawaharLal Nehru Medical College, DMIMSU, Sawangi, wardha, Maharastra, India.

Correspondence: DrSunil Kumar, Associate professor, Department of medicine, JawaharLal Nehru Medical College, DMIMSU, Sawangi, wardha, Maharastra, India. E-mail sunilkumarmed@gmail.com

\section{Case history:}

A 28-year-old man from rural Maharashtra was admitted in medicine department with fever, nasal congestion, blood mixed nasal discharge with redness of eyes, and cough with hemoptysis. He also complained of occasional breathlessness and chest discomfort. He denied any history of pulmonary tuberculosis, bronchial asthma, hypertension and diabetes in past. He was non smoker and nonalcoholic. On general examination he was pale, breathless and having congested eye. On nasal examination he had multiple ulcerative and granulomatous lesions on the septum.(Fig-1) Other examination was within normal limit.His blood examination revealed hemoglobin $8.9 \mathrm{gm} / \mathrm{dl}$, Total Leucocyte Count $14,800 / \mathrm{mm} 3$ with $18 \%$ eosinophils and markedly elevated ESR (114mm in first hour).Liver and renal functions were normal and urinary sediment did not include red blood cells, casts, or proteinuria. His ELISA for HIV was negative.

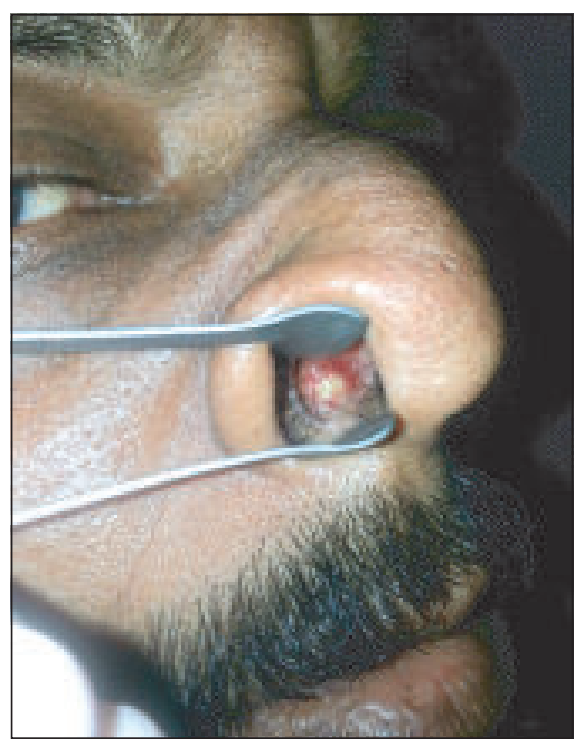

Fig.-1: multiple ulcerative and granulomatous lesions on the septum 
His chest X-ray revealed multiple cavitory lesion in both lung, (fig-2)with subsequent Computerized tomography of chest revealing nodular pulmonary lesions as well as multiple cavititesinboththelungs.(Fig-3)Ultrasound of the abdomen of the patient revealed bilateral raised renal parenchymal echogenecity with normal renal size. Based on the complete radiological findings with relevant clinical and pathological data a possibility of Wegener's Granulomatosis was suggested. This was supported by histopathological examination of nasal granulation tissue which revealed granulomatous inflammation around perivascular area. His Antineutrophil cytoplasmic antibody (C-ANCA) in serum was markedly raised and P- ANCA was negative.Cause of raised eosinophil was uncertain in this case as no other

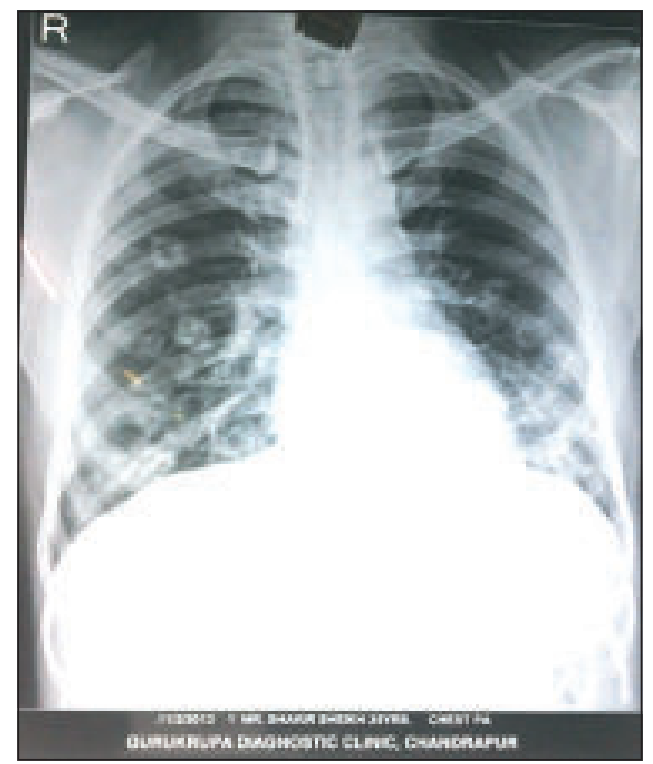

Fig.-2: chest $X$-ray revealed multiple cavitorylesion in both lung

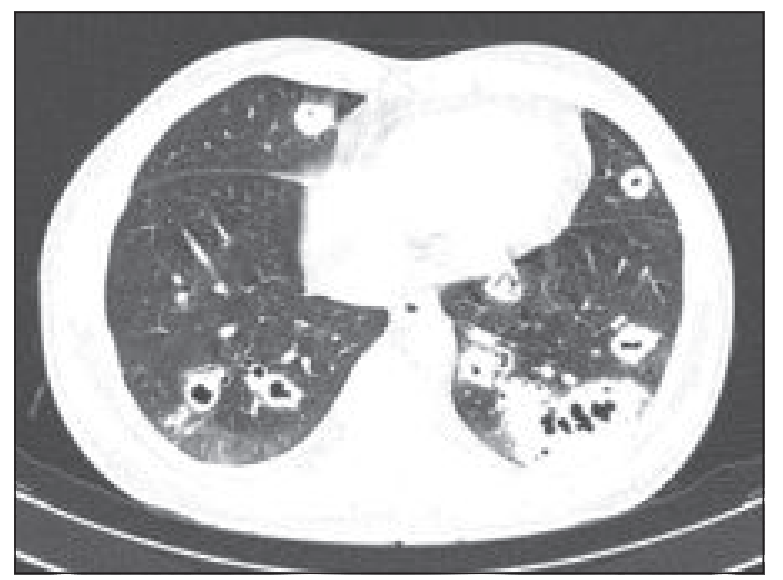

Fig.-3: CT chest reveals nodular pulmonary lesions as well as multiple cavities in both lungs causes of eosinophilia, such as parasites and drugs, were detected. Patientwas put on oral prednisolone $40 \mathrm{mg}$ per day and cyclophosphamide $80 \mathrm{mg}$ per day. He showed marked improvement in clinical symptoms such as cough and breathlessness. He was doing well on follow up.

\section{Discussion:}

WG is a rare autoimmune entity which may occur at any age with mean age of occurrence 40 to 55 years old with equal predisposition to male and female. WG with involvement of the upper respiratory tract and the lungs with renal sparing are frequently seen in women, while the kidneys are involved in the common form frequently seen in men. ${ }^{3}$

In our case rarity was young male with relatively renal sparing. The four criteria of diagnosis defined by the American College of Rheumatology (ACR) for WG are as follows: 1) Oral or nasal ulcers, or purulent bloody flux 2) An abnormal lung X-ray revealing nodules and cavities 3) An abnormal urinary sediment 4) Granulomatous inflammation in the extra vascular region at biopsy. The presence of two or more of these criteria has a sensitivity of $88 \%$ and a specificity of 99\%. [4]PR3-ANCA was positive in this patient and we tentatively diagnosed as WG on the basis of ACR criteria. However, because of eosinophilia in peripheral blood possibility of CSS was also kept. But there was no increased IgE level in the peripheral blood, no peripheral neuropathy, and no history of asthma. In our patient PR3-ANCA was present in high titer and percentage of WG patients with PR3-ANCA ismuch higher than that of CSS patients. Also this patient had granulomatous inflammation of thenasal mucosa, which is a rare finding in patients with CSS. Till now only four cases of WG with peripheral eosinophilia (more than 1,500/1) have been reported to date. [5-8] According to the Hammanrich criteria for CSS, a peripheralblood eosinophil count of more than 1,500/1 is suggestiveof CSS. ${ }^{5}$ However, the four previous cases and the presentpatient were considered as WG with eosinophilia in view ofthe involved organs and theexistence of PR3-ANCA, eventhough their peripheral blood eosinophil count was higherthanthatlevel. Computerized tomography of chest commonly shows discrete focal opacities that vary in size and appearance from diffuse consolidation to nodular masses which cavitate in at least $25 \%$. [9]Cavity walls vary in thickness considerably, but tend to become thinner with time.

Limited forms of WG sparing the kidney are rare. Usually severe progressive necrotizing glomerulonephritis occurs resulting in rapid deterioration of renal function. Large echogenic kidneys on ultrasound without specific Doppler abnormalities are typical early findings. [5]WG should be considered in cases presenting with nasal disease and 
associated chest signs and symptoms and early diagnosis and prompt treatment should be performed.

\section{Conflict of Interest : None}

\section{References:}

1. Carol A. Langford, Anthony s fauci: the vasculitis syndrome, granulomatosis with polyangitis (wegner's). In: Longo DL, Fauci AS, Kasper DL, Hauser SL,JamesonJL ed. Harrison's principles of internal medicine,18th edn. New York: McGraw-Hill Inc, 2012. p. 2789.

2. Seaton A. Pulmonary lymphocytic angitis and granulomatosis. In, Seaton A (ed). Crofton and Douglas's Respiratory Diseases. 5th edition, London, Blackwell Science Inc, 2000;1063

3. Verma S R. Case Report- Wegener's Granulomatosis in a Young Adult Patient. Webmed Central RADIOLOGY 2011;2(9):WMC002210.
4. Leavitt RY, Fauci AS, Bloch DA, Michel BA, HunderGG, Arend WP et al. The American College of Rheumatology 1990 criteria for the classification of Wegener's granulomatosis. Arthritis Rheum 1990;33:1101-7.

5. Shoda H, Kanda H, Tanaka R, Komagata Y,Misaki Y, Yamamoto K. Wegener's Granulomatosis with Eosinophilia. Internal Medicine 44: 2005.750-753.

6. Potter MB, Fincher RK, Finger DR. Eosinophilia in Wegener'sGranulomatosis. Chest 116: 1480-1483, 1999.

7. Lane SE, Watts RA, Baker HW, Scott DG. Evaluation of the Sørensondiagnostic criteria in the classification of systemic vasculitis.Rheumatology (Oxford) 41: 1138-1141, 2002.

8. Kamali S, Kasapoglu E, Aktürk F, et al. Eosinophilia and hyperimmunoglobulinemiaE as the presenting manifestations of Wegener's granulomatosis. ClinRheumatol22: 333-335, 2003.

9. Lohrmann C, Uhl M, Kotter E, Burger D, GhanemN,Langer M. Pulmonary manifestations of Wegener granulomatosis: CT findings in 57 patients and areview of theliterature. EurJRadiol.2005;53:471-7. 